\title{
Physics prospects and plan of SuperKEKB/Belle II
}

\author{
Anatoly Sokolov ${ }^{1}$ \\ Institute for High Energy Physics \\ Nauka square 1, 142281 Protvino, Russia \\ E-mail: sokolov alihep.ru
}

Belle II experiment at the SuperKEKB collider is a major upgrade of the Belle experiment at the KEKB asymmetric $e^{+} e^{-}$collider at the KEK. The experiment will focus on the search for physics beyond the Standard Model using indirect probes in the flavor sector. High precision measurements of $B$, charm and tau lepton decays complement direct searches at the highest energies and provide crucial, new information. In this talk the physics prospects and the present status of the upgrade are presented.

The XXI International Workshop High Energy Physics and Quantum Field Theory

June 23 - June 30, 2013

Saint Petersburg Area, Russia

\footnotetext{
${ }^{1}$ Speaker

on behalf of the Belle II Collaboration
} 


\section{Introduction}

The B-factory experiments Belle [1] at the KEKB accelerator at KEK, and BaBar [2] at the PEPII accelerator at SLAC, have not only successfully confirmed the Kobayashi-Maskawa mechanism of CP violation (CPV) in the Standard Model (SM) [3], but also carried out a rich physics program covering B-physics, charm physics, tau physics. Altogether, about $1.5 \mathrm{ab}^{-1}$ data in the vicinity of the $\Upsilon(4 \mathrm{~S})$ resonance were accumulated, corresponding to over 1 billion $B \bar{B}$ pairs. So far most measurements, not only at the B-factories, are in a good agreement with the Standard Model predictions. However, there are unresolved issues in the SM, like the lack of sufficient CP violation to explain the baryon asymmetry in the Universe, that require New Physics (NP) processes. The search for NP effects in high precision measurement is the mission of the Belle II experiment [4]. It will be located at the upgraded KEKB accelerator, SuperKEKB, and record $e^{+} e^{-}$collision data with an upgraded Belle detector. The aim is to accumulate $50 \mathrm{ab}^{-1}$, corresponding to about 55 billion $B \bar{B}$ pairs and about 47 billion $\tau^{+} \tau^{-}$events, by the year 2022 .

\section{Belle II physics}

B-factories demonstrated the effectiveness of flavour physics (i.e. physics of transitions between the three generations of standard model fermions) in constraining New Physics. It was shown that SM is a valid effective theory at the current energy scale. LHC started to examine $\mathrm{SM}$ at higher energies. In order for the flavour physics to be useful in the LHC era, B-factories can make complimentary search for NP. For it the indirect probes can be used, which can explore regions of the parameter space are not covered at the LHC, or provide additional information on new phenomena possibly discovered at the highest energies.

The precision of various flavour measurements must be significantly improved, both in terms of experimental reach and understanding of theoretical uncertainty. A precision which is enough to be relevant for $\mathrm{TeV}$ processes depends on the NP flavor changing couplings. If minimally flavour violating NP has couplings to SM fermions comparable to weak gauge couplings, the present results from B-factories allow for masses of NP particles below $\sim 100$ $\mathrm{GeV}$. After completion of the Belle II program this limit would be pushed to $\sim 1000 \mathrm{GeV}$. However it can be increased in a case of enhanced couplings.

The flavour physics processes that proceed through flavour changing neutral currents are useful for NP searches. These processes are loop suppressed in SM, and hence NP contributions are easier to detect here than in charged flavour changing transitions that occur at the tree level in SM.

Another powerful probe of NP effects is the measurement of $\mathrm{CP}$ violating observables. Extensions of SM lead to new sources of CP-odd phases and/or new sources of flavor breaking.

The large increase in statistics will provide a step change in NP sensitivity studies. The corresponding program will include not only much more precise studies of NP-sensitive observables for which initial studies have already been carried out (e.g., $b \rightarrow s g, b \rightarrow s \gamma$, and $b \rightarrow s l^{+} l^{-}$penguin dominated processes) but also channels which, at their SM expectations, are beyond the capabilities of current experiments $(b \rightarrow d$ penguin dominated processes, $b \rightarrow s v \bar{v}$ decays). For some channels with very small SM expectations positive searches would provide unambiguous NP signals (e.g., lepton flavor violating $\tau$ decays, $C P$ violation in charm mixing, and/or decays, $b \rightarrow d d \bar{s}$ decays).

$e^{+} e^{-}$machine provides a much cleaner environment than hadron collider, which is essential for important observables that involve $\gamma$ 's, $\pi^{0}$ 's, $K_{L}^{0}$ 's or neutrinos in the final states. 
At the $\Upsilon(4 S)$ resonance, the $B \bar{B}$ pair is produced near the energy threshold and there are no associated particles. This means that by reconstructing the full energy-momentum vector of a $B$ $(\bar{B})$ meson from its daughter particles (the full reconstruction technique), one can infer the missing momentum in the decay of the other $\bar{B}(B)$ meson. This technique is essential for the measurement of channels including neutrino(s) in the final state. The measurement of the CKM element $\left|V_{u b}\right|$ through the semi-leptonic decay $b \rightarrow u l \bar{v}$, the search for a charged Higgs effect in $B \rightarrow D \tau \bar{v}_{\tau}$, and measurements of $B \rightarrow K v \bar{v}, B \rightarrow \tau v_{\tau}$ fall in this class. Therefore, there is no doubt that SuperKEKB is complementary to the future experiments at the hadron machines from both, the motivational and the experimental aspect.

Below there are discussed some processes which will be studied at Belle II.

\subsection{New Source of CP violation: $b \rightarrow s q \bar{q}$}

One of the most important channels to search for NP in B physics are the $b \rightarrow s$ transitions. The largest NP effect could come from the penguin diagrams which can be studied in the timedependent $\mathrm{CP}$ asymmetries in $B \rightarrow \varphi K_{S}, B \rightarrow \eta^{\prime} K_{S}$ modes. In the SM the time-dependent CP asymmetries in these modes are similar to the one in $B \rightarrow J / \psi K_{S}$. The $B-\bar{B}$ oscillation part is the same and the decay parts (penguin diagram for the former and tree diagram for the later) do not contain any $\mathrm{CP}$ violating phase. NP can give a contribution to a $\mathrm{CP}$ violating phase for $B \rightarrow$ $\varphi K_{S}, B \rightarrow \eta^{\prime} K_{S}$ decays and we can have $S_{b s} \neq S_{b c}$ for indirect CP parameters. Also the direct $\mathrm{CPV}$ can be not equal to zero. (The decay amplitude of $B \rightarrow J / \psi K_{S}$ is mainly due to the tree level diagram and does not changed by NP). To extract NP it is necessary to measure a deviation of the $\phi_{1}$ angle in $B \rightarrow \varphi K_{S}, B \rightarrow \eta^{\prime} K_{S}$ decays from the SM reference value.

The $\phi_{1}$ angle measured at Belle and BaBar in $b \rightarrow s$ decays. There is no clear deviation from the SM seen in all modes. New CPV effect could be seen with much larger data.

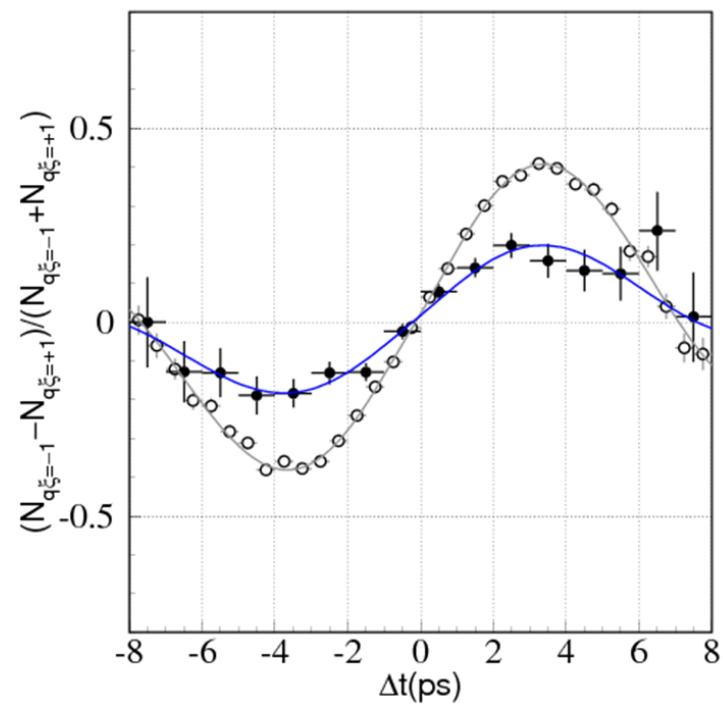

Fig. 1. Raw asymmetries for $B \rightarrow \varphi K_{S}$ (closed circles) and $B \rightarrow J / \psi K_{S}$ (open circles) at $50 \mathrm{ab}^{-1}$. 2010 world average values $S_{\varphi K s}=+0.39$ and $A_{\varphi K s}=0$ are used as input values for $B^{0} \rightarrow \varphi K^{0}$.

At Fig. 1 is shown the comparison of time-dependent $\mathrm{CP}$ asymmetries in $B \rightarrow \varphi K_{S}$ decay with the $B \rightarrow J / \psi K_{S}$ one with the luminosity of $50 \mathrm{ab}^{-1}$. A difference of the timedependent $\mathrm{CP}$ asymmetry amplitude in $B \rightarrow \varphi K_{S}$ decay will say about deviation of the $\phi_{1}$ angle from the SM value. The shift of this curve will say about presence of the direct CPV in this 
decay. At Fig. 2 is shown the dependence of the indirect CPV parameter $S_{C P}$ difference $\Delta S$ measured in different processes from the integrated luminosity. Prospects for the $\Delta S$ uncertainty is of about 0.02 for $50 \mathrm{ab}^{-1}$. The corresponding uncertainty is enough to feel NP.

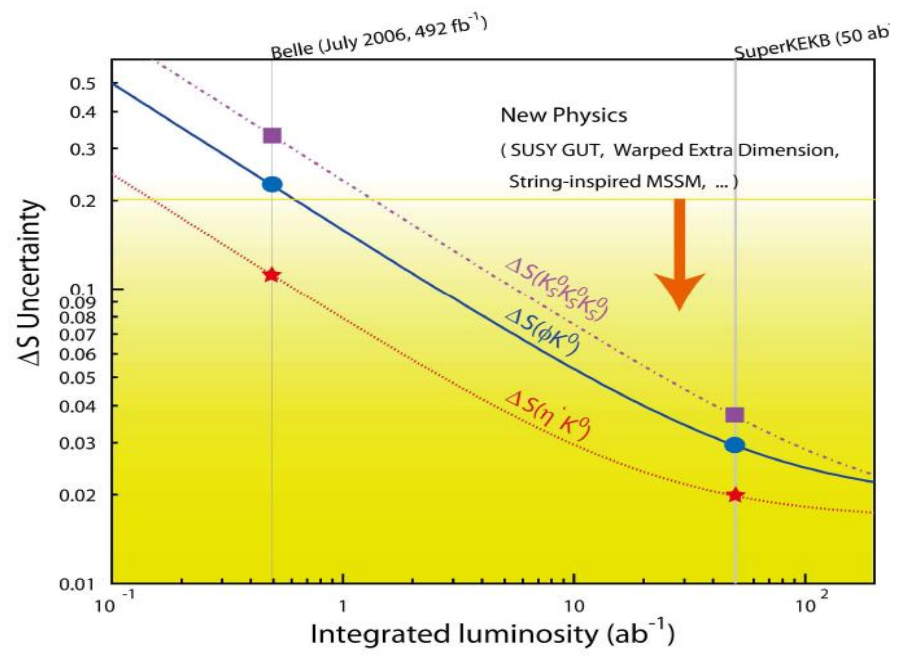

Fig. 2. Expected total errors on $\Delta S$ as a function of integrated luminosity.

\subsection{Radiative B decays}

Radiative and electroweak decays from the $b \rightarrow s(d)$ transitions with the $\gamma$ radiation ( $b \rightarrow s \gamma, b \rightarrow d \gamma, b \rightarrow s l^{+} l^{-}$) starts at the one loop order. They are sensitive to the New Physics (see the diagrams at Fig. 3). Deviations from SM predictions could be looked for in the following radiative and electroweak decays studies:

- precise measurement of inclusive $B \rightarrow X_{s} \gamma$ branching fraction,

- measurement of inclusive $B \rightarrow X_{d} \gamma$ branching fraction,

- direct CP violation in the $B \rightarrow X_{s} \gamma, B \rightarrow K^{*} \gamma, B \rightarrow X_{s} l^{+} l^{-}$and so on decays,

- time-dependent CP violation in $B \rightarrow K^{*} \gamma, B \rightarrow \rho \gamma$ and related modes,

- measurement of photon polarization with photon conversion,

- measurement of the forward-backward asymmetry and $q^{2}$ distribution of $B \rightarrow K^{*} l^{+} l^{-}$and $B \rightarrow X_{s} l^{+} l^{\prime}$,

- lepton flavor dependence of $b \rightarrow s l^{+} l$.
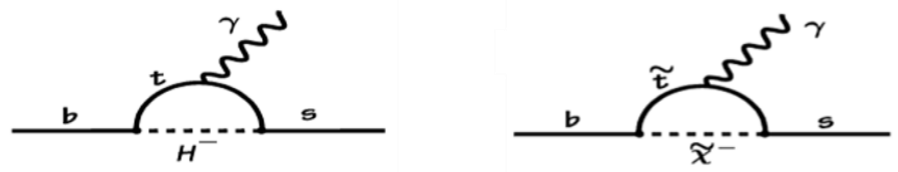

Fig. 3. NP diagrams of the radiative $b \rightarrow s \gamma$ decays.

\subsubsection{Exclusive $b \rightarrow s \gamma$ decays}

The new physics can give a contribution to the $B \rightarrow K^{*} \gamma$ decay. The final state in this decay is self-charge conjugate $\left(B^{0} \rightarrow K^{*^{0}} \gamma \rightarrow K_{S} \pi^{0} \gamma\right)$. It's possible to see CP violation in this decay by measuring the time-dependent $\mathrm{CP}$ asymmetry. The effect of a new physics can give a contribution to the CPV parameters. 
Within the Standard Model, the photon emitted in this decay is predominantly polarized. A flip of photon polarization is suppressed by the quark mass ratio $2 m_{s} / m_{b}$ [5]. The Standard Model predicts a small time-dependent CP asymmetry in this decay. A significant deviation from the small SM prediction could indicate new physics. For example left-right symmetric model give much higher value of indirect CPV parameter $S_{C P} \sim 0.5[5,6]$.

Belle and $\mathrm{BaBar}$ have performed time-dependent $\mathrm{CP}$ asymmetry measurement for $B^{0} \rightarrow$ $K_{S} \pi^{0} \gamma$ decay. The average results for the indirect and direct CP violating parameters are $S_{C P}=$ $0.15 \pm 0.20, A_{C P}=-0.07 \pm 0.12$. The estimated uncertainty of the $S_{C P}$ parameter with the Belle II luminosity of $50 \mathrm{ab}^{-1}$ is 0.03 . Fig. 4 shows the comparison of the measured $S_{C P}$ and $C_{C P}\left(=-A_{C P}\right)$ parameters with the expectation from Belle II experiment. The measured in the $B^{0} \rightarrow K^{*^{0}} \gamma$ decay parameters of CPV in a combination with precise measurements of $\operatorname{Br}\left(B_{s} \rightarrow \mu^{+} \mu\right)$ at the LHCb can give improved constraints on the parameters of MSSM.

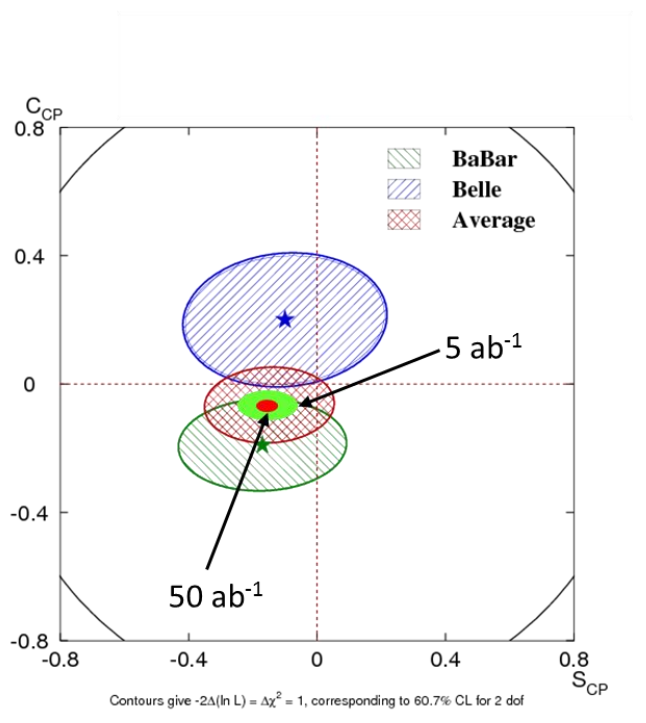

Fig. 4. Current world average values and the expected precision with $50 \mathrm{ab}^{-1}$ of data on indirect $\left(S_{C P}\right)$ and direct $\left(C_{C P}\right) \mathrm{CPV}$ in $B^{0} \rightarrow K_{S} \pi^{0} \gamma$.

\subsubsection{Inclusive $B \rightarrow X_{s} \gamma$ decay}

The branching fraction of the inclusive $B \rightarrow X_{s} \gamma$ decay is sensitive to charged Higgs contribution. If we will see the deviation of the measured branching fraction from the SM value then it's possible to estimate contribution and parameters of NP.

It is hard to study this process because we have a huge background. The background from continuum is of two orders of magnitude higher of the signal. To reduce this background events with a high gamma energy are considered. The advantage of B-factories that it's possible to tag the another $B$ in the event. This procedure reduces the continuum background considerably and improves signal/background ratio. In the opposite hemisphere only gamma is reconstructed.

The branching fraction of the inclusive $B \rightarrow X_{s} \gamma$ decay has been measured by the Belle experiment. An accuracy of this measurement is of about $12 \%$ with the systematics domination. The stronger tagging (e.g. full reconstruction of the another $B$ ) can reduce the systematics. So to measure the branching fraction of the inclusive $B \rightarrow X_{s} \gamma$ decay with a higher precision a large statistics of the Belle II experiment is necessary. 
$2.3 B \rightarrow \tau v_{\tau}$

The purely leptonic decays $B^{+} \rightarrow l^{+} v_{l}$ are suppressed in the Standard Model due to the wrong helicity leptons in the final state and the corresponding branching is low. Now, only the $B^{+} \rightarrow \tau^{+} v_{\tau}$ mode can be measured.

In the Standard Model, the purely leptonic decay $\left(B^{+} \rightarrow \tau^{+} v_{\tau}\right)$ proceeds via annihilation of $b$ and $u$ quarks to a $W^{+}$boson. It provides a determination of the product of the $B$ meson decay constant $f_{B}$ and the Cabibbo-Kobayashi-Maskawa (CKM) matrix element $\left|V_{u b}\right|$. The decay constant $f_{B}$ can be easy calculated on the lattice QCD. The element $\left|V_{u b}\right|$ can be determined from independent measurements. Physics beyond the Standard Model, for example two-Higgs doublet model, could modify $B\left(B^{+} \rightarrow \tau^{+} v_{\tau}\right)$ through the introduction of a charged Higgs boson [7].

$$
\Gamma\left(B^{+} \rightarrow \tau^{+} v_{\tau}\right)=\Gamma_{S M}\left(B^{+} \rightarrow \tau^{+} v_{\tau}\right) \cdot\left(1-\frac{m_{B}^{2}}{m_{H}^{2}} \tan ^{2} \beta\right)^{2} .
$$

The experimental measurement of this decay is difficult due to the two missing neutrinos. The $B$ tagging technique is used in this measurement. The signal from the $B^{+} \rightarrow \tau^{+} v_{\tau}$ decay is looking for in the rest of the event. The signature is ( 1 track + invisible particles). The main discriminating variable on the signal side is the remaining energy in the calorimeter, not associated with any charged track or photon. We have a signal at $\mathrm{E}_{\mathrm{ECL}}=0$. Belle and $\mathrm{BaBar}$ have measured this branching.

The $5 \sigma$ discovery region for the integrated luminosity of $50 \mathrm{ab}^{-1}$ for the parameters of two-Higgs doublet model shown at the Fig. 5 (red region). Here are also presented the similar region for LEP (yellow), Tevatron (grey), current region for B factories (green). The branching of the pure leptonic $B$ decay can change also in another models beyond SM. These model also will be examined.

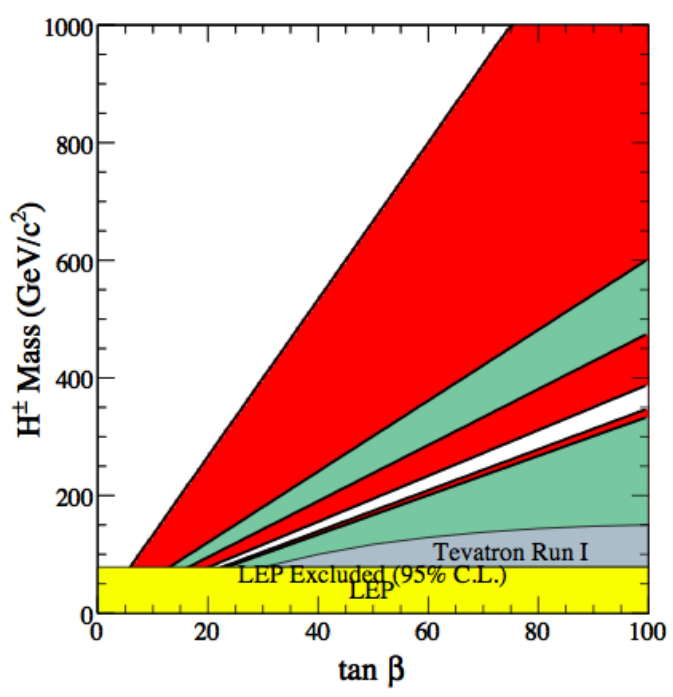

Fig. 5. The red area shows the $5 \sigma$ discovery reach for the charged Higgs boson using the decay mode $B^{+} \rightarrow \tau^{+} v_{\tau}$ and $50 \mathrm{ab}^{-1}$ of $\Upsilon(4 \mathrm{~S})$ data (from Ref. [8]). 
$2.4 b \rightarrow h v \bar{v}$

In the Standard Model the rare decay $b \rightarrow s v \bar{v}$ proceeds at the one-loop level through penguin and box diagrams (see Fig. 6). The branching fractions of these channels are of about $10^{-6}$.
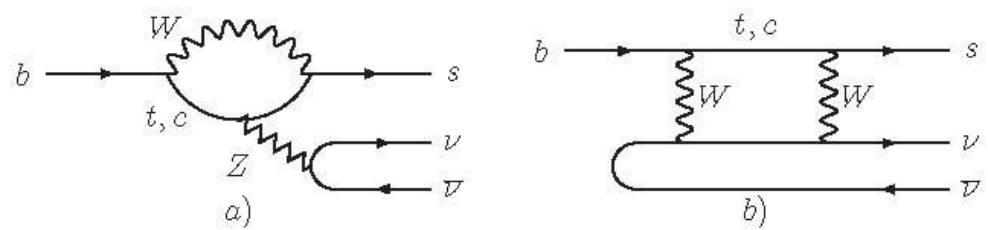

Fig. 6. Penguin (a) and box (b) diagram of the $b \rightarrow s v \bar{\nu}$ decay.

New physics may contribute to this decay mode. For example in models with righthanded currents these decay modes will increase. At Belle II the branching fraction of these decays can be measured with a precision of $30 \%$. This measurement can put limits on the righthanded currents.

The experimental measurement of $b \rightarrow s v \bar{v}$ is hard due to two missing neutrinos. To extract the signal events the tagging technique could be used. Here events with one tagged $B$ meson are selected and in the opposite hemisphere we search for a signal. It should contain $K$ or $K^{*}$ and have a zero calorimetric energy. For this study it is important to have a detector with a high hermeticity and high ECL performance.

At Fig.7 there are shown constraints on the NP right-handed couplings arising from the measurements of $\operatorname{Br}\left(B \rightarrow K^{(*)} v \bar{v}\right)$ with $50 \mathrm{ab}^{-1}$. Green lines represent current exclusion limits from the existing upper limits of the branching ratios for the two decays. Red lines are the constraints arising from the measurements with the precision $\pm 30 \%$, and blue lines denote the theoretical accuracy (from [9]).

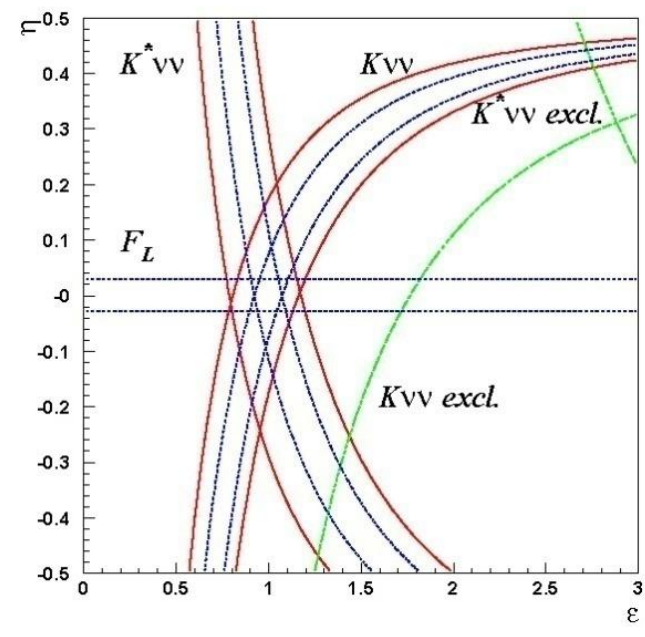

Fig. 7. Constraints on the NP right-handed couplings arising from the measurements of $\operatorname{Br}\left(B \rightarrow K^{(*)} v \bar{v}\right)$ with $50 \mathrm{ab}^{-1}$ (see text).

\subsection{Lepton flavour violation}

The SuperKEKB will be not only a $B$ meson factory, but also tau- and charm- factory. One of the most important physics targets of the Belle II experiment will be a study of a lepton flavor violation (LFV) in tau decays. Lepton flavor conservation in the Standard Model is 
associated with massless neutrinos. Because neutrinos have a finite but tiny masses charged lepton flavor violating processes are strongly suppressed and beyond experimental sensitivity. For example the branching fraction of the $\tau \rightarrow \mu \gamma$ decay is $B r(\tau \rightarrow \mu \gamma) \sim 10^{-49}$ [10] in SM. Any signal lepton flavor violation is an unambiguous sign of New Physics. In many extensions of SM the LFV decays are significantly enhanced. The branching fractions of the $\tau \rightarrow l \gamma$ (SUSY model), $\tau \rightarrow \mu \mu \mu, \tau \rightarrow \mu \eta$ (a little Higgs model) decays are high enough for the experimental study. At Fig. 8 is presented the experimental sensitivity for a study of LFV in tau decays. Here is presented the CLEO results, the B-factory results and expected sensitivity for Belle II. We can see that the last sensitivity will be improved by one order of magnitude in comparison with Belle. This enable to examine many models beyond SM.

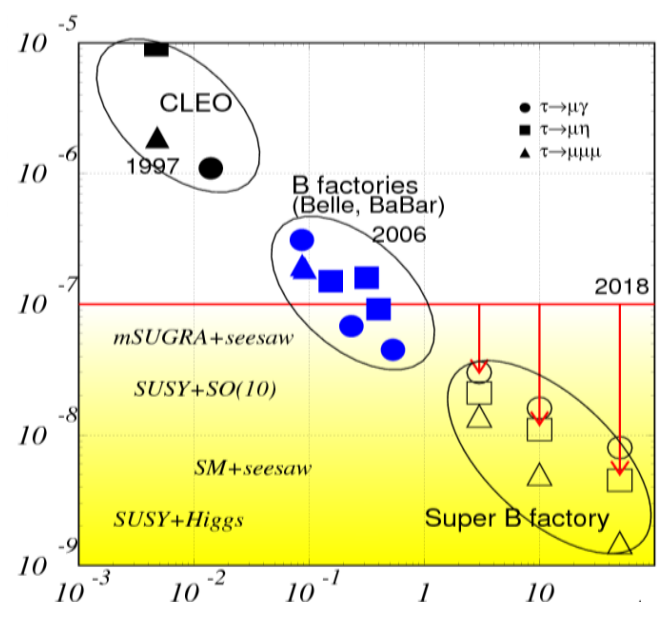

Fig. 8. Achieved sensitivity on the branching ratio of the LFV searches and the prospects in the Belle II experiment.

\section{Accelerator}

SuperKEKB is built by upgrading the existing KEKB machine, which is an asymmetric energy collider consisting of an $8 \mathrm{GeV}$ electron (HER) and a $3.5 \mathrm{GeV}$ positron ring (LER) The SuperKEKB $e^{+} e^{-}$collider is designed to reach an instantaneous luminosity of $8 \times 10^{35} \mathrm{~cm}^{-2} \mathrm{~s}^{-1}$, forty times higher than the KEKB record luminosity. To go beyond the KEKB performance, there are basically two strategies which can be discussed by considering the expression for the instantaneous luminosity,

$$
L=\frac{\gamma_{ \pm}}{2 e r_{e}}\left(1+\frac{\sigma_{y}^{*}}{\sigma_{x}^{*}}\right) \frac{I_{ \pm} \xi_{y, \pm}}{\beta_{y, \pm}^{*}} \frac{R_{L}}{R_{\xi_{y}}} .
$$

Here, $\gamma$ is the Lorentz factor, $\sigma_{x}^{*}$ and $\sigma_{y}^{*}$ are the horizontal and vertical beam sizes at the interaction point (IP), $I$ the beam current, $\beta_{y, \pm}^{*}$ is the vertical beta function at the IP, $\xi_{y, \pm}$ is the beam-beam parameter, and $R_{L} / R_{\xi_{y}}$ is a reduction factor reflecting the finite crossing angle of the beams. The subscript \pm refers to the product of the corresponding quantities for the low energy positron and high energy electron beams.

Only few parameters in this expression can be significantly altered: To increase luminosity, one can increase the stored currents $I_{+}$and $I$-, which corresponds the high current strategy. The main disadvantage of this solution is the need to substantially increase the power of the accelerating cavities, leading to high operation costs. High currents also imply high levels 
of background in the experiment from beam-beam and beam-gas scattering. The high energy loss due to synchrotron radiation causes machine damage and backgrounds in the detector. Mainly because of high operation costs, this option was dropped.

The second strategy is the low emittance scheme [11], which has been adopted as the baseline for SuperKEKB [4]. It foresees a drastic squeezing of the IP beam size $\sigma_{y}^{*}$ from 1.1 $\mu \mathrm{m}$ at KEKB to $0.08 \mu \mathrm{m}$ at SuperKEKB. Due to this extremely small vertical beam size, this option is also referred to as nano-beam scheme. Low emittance means that the average spread of particle positions and momenta must be extremely small, to make particles actually collide in this small volume. A luminosity of $8 \times 10^{35} \mathrm{~cm}^{-2} \mathrm{~s}^{-1}$ can be achieved with only a moderate increase in beam currents compared to KEKB.

The upgrade from KEKB to SuperKEKB requires the replacement of the long dipoles with shorter ones, a new interaction region with new final focusing quadrupoles near the IP, and a redesign of the HER and LER lattices to squeeze the emittance.

By new quadrupole magnets in the interaction region the beta functions are reduced in $y$ direction from $5.9 \mathrm{~mm}$ to $0.27 / 0.31 \mathrm{~mm}$ for HER/LER, and in $\mathrm{x}$ direction from $120 \mathrm{~cm}$ to $3.2 / 2.5 \mathrm{~cm}$.

Since the beam-beam parameter is proportional to $\sqrt{\beta^{*} / \varepsilon}$ the emittance $\varepsilon$ has to be reduced to keep the beam-beam parameter at a similar level as at KEKB. A reduction of the emittance from 18/24 $\mathrm{nm}$ to $3.2 / 5.0 \mathrm{~nm}$ is obtained by installing a new electron source and a new damping ring, in addition to a redesign of the HER and LER lattices. The last contribution to the luminosity gain comes from higher beam currents. They are increased from 1.6/1.2 A to 3.6/2.6 A.

The higher luminosity also leads to higher background levels. The effect of background source, like radiative Bhabha scattering, Touschek scattering, and beam-gas interactions, on the detector performance is assessed in detailed simulation studies. A further consequence of the design for a luminosity of $8 \times 10^{35} \mathrm{~cm}^{-2} \mathrm{~s}^{-1}$ is the reduction of the beam energy asymmetry from $3.6 / 8 \mathrm{GeV}$ to $4 / 7 \mathrm{GeV}$ and an enlargement of the crossing angle from $22 \mathrm{mrad}$ to $83 \mathrm{mrad}$. A summary of the main accelerator parameters for KEKB and SuperKEKB can be found in Tab. 1.

\begin{tabular}{|c|c|c|c|c|}
\hline \multirow[t]{2}{*}{ Parameter } & \multicolumn{2}{|c|}{ KEKB } & \multicolumn{2}{|c|}{ SuperKEKB } \\
\hline & LER & HER & LER & HER \\
\hline Beam energy Ebeam $(\mathrm{GeV})$ & 3.5 & 8 & 4 & 7 \\
\hline Half crossing angle $\phi(\mathrm{mrad})$ & \multicolumn{2}{|c|}{11} & \multicolumn{2}{|c|}{41.7} \\
\hline Horizontal emittance $\varepsilon x(\mathrm{~nm})$ & 18 & 24 & 3.2 & 5.0 \\
\hline Emittance ratio $\kappa(\%)$ & 0.88 & 0.66 & 0.27 & 0.25 \\
\hline Horizontal beta function at IP $\beta_{x}^{*}(\mathrm{~mm})$ & \multicolumn{2}{|c|}{1200} & 32 & 25 \\
\hline Vertical beta function at IP $\beta_{y}^{*}(\mathrm{~mm})$ & \multicolumn{2}{|c|}{5.9} & 0.27 & 0.31 \\
\hline Beam currents Ibeam $(\mathrm{A})$ & 1.64 & 1.19 & 3.60 & 2.60 \\
\hline Beam-beam parameter $\xi_{y}$ & 0.129 & 0.090 & 0.0886 & 0.0830 \\
\hline Luminosity $L\left(10^{34} \mathrm{~cm}^{-2} \mathrm{~s}^{-1}\right)$ & \multicolumn{2}{|c|}{2.1} & \multicolumn{2}{|c|}{80} \\
\hline
\end{tabular}

Table 1. Machine parameters of the present KEKB collider and of SuperKEKB (from Ref. [4]).

The ground-breaking ceremony at KEK on November 18, 2011, was the formal start of the SuperKEKB project. The first new LER dipole magnets were installed in the tunnel on 
February 7, 2012. The construction of the new damping ring has started in 2012 as well. Commissioning of SuperKEKB is planned for the year 2015. Expecting that it will reach its design performance in the year 2020, an integrated luminosity of $50 \mathrm{ab}^{-1}$ will be collected until 2022 (Fig. 9).

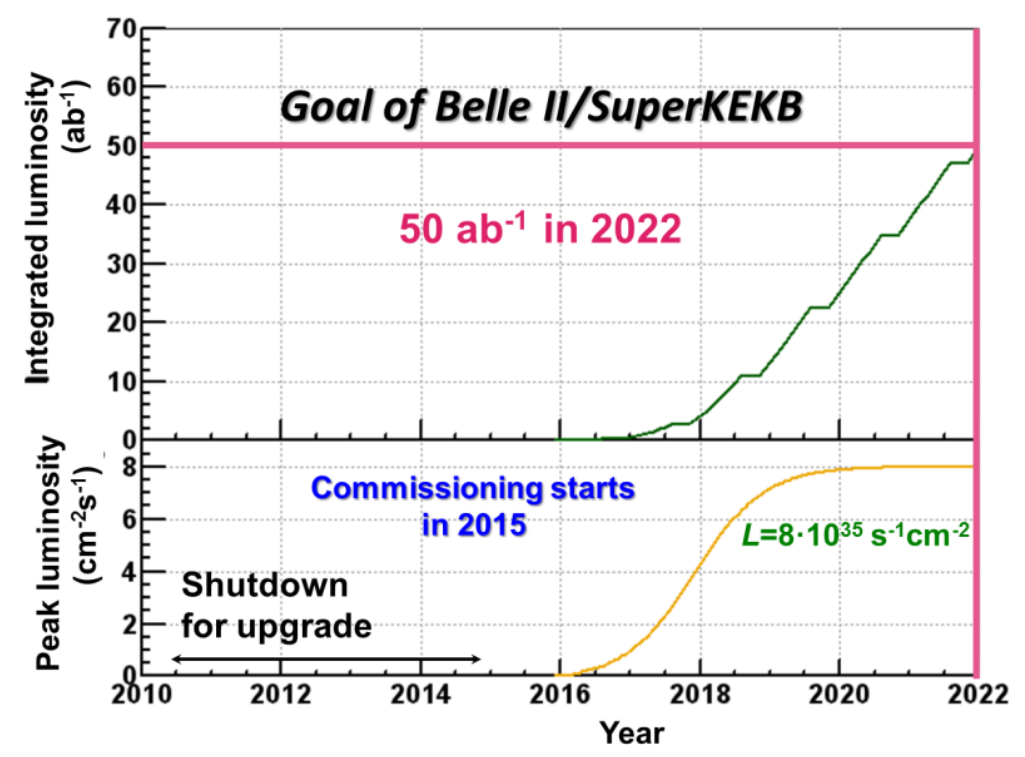

Fig. 9. Projected SuperKEKB luminosity.

\section{Detector}

Because of the increased level of background, the Belle II detector has to cope with higher occupancy and radiation damage than the Belle detector. Furthermore the increased event rate puts high demands on trigger, data acquisition, and computing. To be able to operate at the conditions at the SuperKEKB collider, the components of the Belle detector are either upgraded or replaced by new ones. The design goal of Belle II is to maintain or even improve the data quality with respect to Belle in the high luminosity environment. Fig. 10 shows the components of the Belle II detector and their main features. A detailed description of the detector can be found in Ref. [4].

The innermost part of the tracking system consists of two layers of silicon pixel sensors (PXD) based on the DEPFET technology. DEPFET is a novel technology originally developed for vertex detection at the International Linear Collider (ILC), which has now reached a high level of maturity [12]. For Belle II, the use of very thin $(75 \mu \mathrm{m})$ detectors with a pitch around $38 \times 50 \mu \mathrm{m}^{2}$ is planned. It is surrounded by four layers of double sided silicon strip detectors (SVD). With the excellent spatial resolution of the PXD an impact parameter resolution in beam direction of $\sim 20 \mu \mathrm{m}$ can be achieved leading to an improved determination of the vertex position. The larger outer radius of the SVD $(13.5 \mathrm{~cm})$ compared to Belle gives an increase in efficiency of about $30 \%$ for the reconstruction of $K_{S} \rightarrow \pi^{+} \pi^{-}$decays inside the SVD. A precise measurement of the momentum of charged tracks is provided by the central drift chamber (CDC). Improvements in the momentum resolution and the particle identification via $d E / d x$ compared to the Belle CDC are achieved by a larger outer radius. A smaller cell size in the inner part increases the background tolerance. New electronics reduces the dead time from $\sim 1 \mu$ s to $200 \mathrm{~ns}$. 


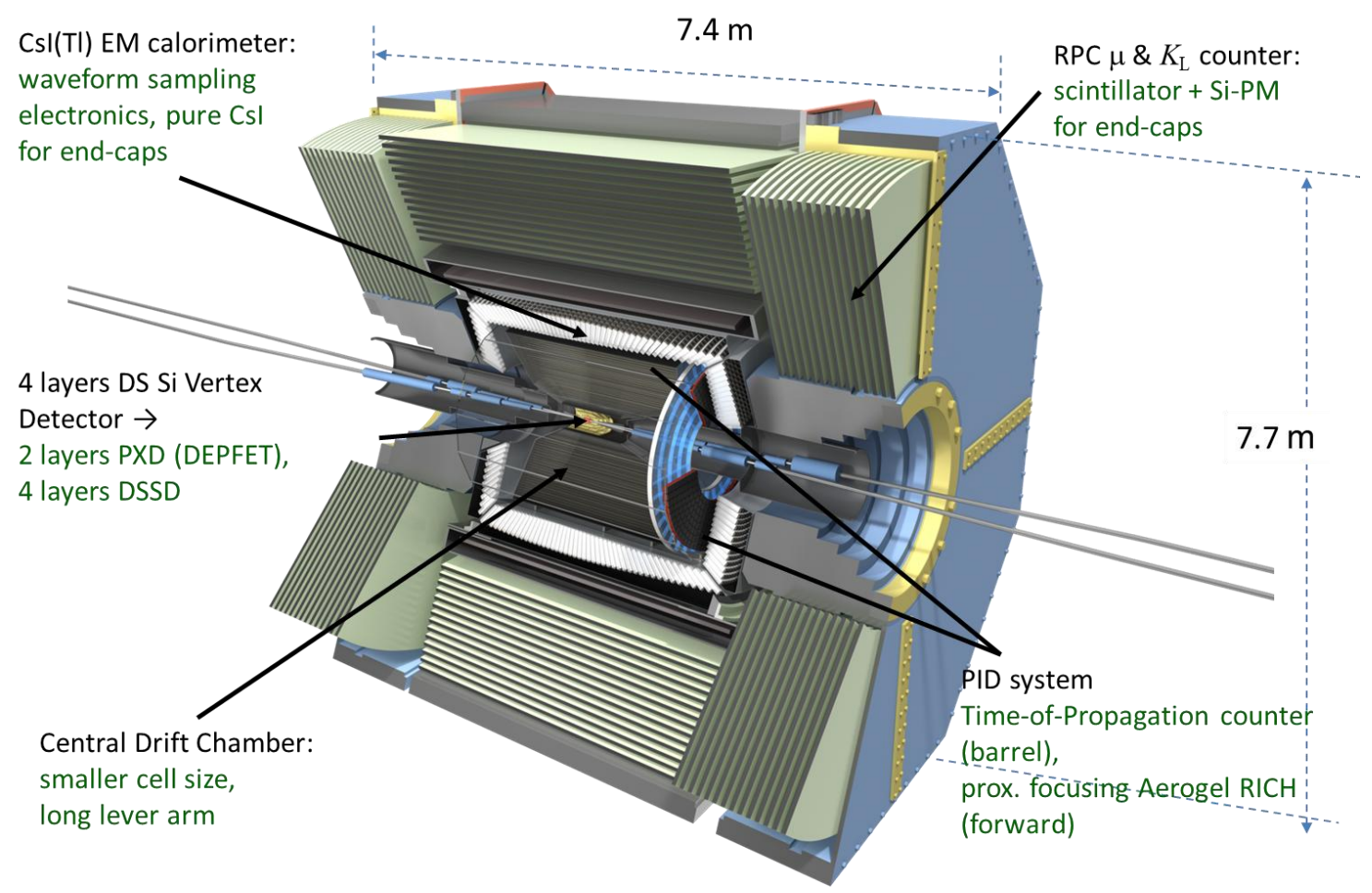

Fig. 10. The Belle II detector.

For the identification of charged hadrons, the time-of- flight detector at Belle is replaced by a time-of-propagation counter (TOP). The TOP consists of a quartz radiator readout by MCP-PMTs in the backward region. Cherenkov photons generated in the radiator are internally reflected and propagate to the PMTs. There, arriving photons are detected in two spatial dimensions and the time of propagation is measured. This three-dimensional information provides improved K/p separation compared to Belle. Another improvement is the new forward endcap particle identification system. The forward region is instrumented with new RICH detectors (ARICH) using aerogel layers with different refractive index to generate Cherenkov rings with the same radius for each layer.

While the CsI(Tl) crystals in the barrel part of the Belle electromagnetic calorimeter (ECL) will be reused for Belle II, the endcaps will be equipped with pure CsI crystals which are faster and more radiation tolerant. To improve the signal to background separation under the higher background conditions at SuperKEKB, the electronics will be upgraded to enable a wave form sampling. Muons and $K_{L}$ mesons are identified by resistive plate chambers in the outer part of the Belle detector (KLM). For Belle II the endcap regions and the inner layers of the barrel region will be upgraded with scintillator strips to cope with the higher background rates.

\section{Summary}

The investigation of physics beyond the Standard Model using indirect probes in the flavour sector complements searches at high energies and provides crucial, new information. This is the physics case of the KEK Super B-factory, consisting of the SuperKEKB machine and the Belle II detector, constructed by upgrading the present B factory.

The upgrade strategy chosen for the machine is the low emittance option, in which the high luminosities are achieved by drastically squeezing the beam size at the interaction point. 
Also the detector undergoes a major upgrade with the replacement of many sub-systems. The Belle II detector will have improved rate tolerance and better hermeticity compared to Belle.

The upgrade project was formally started in November 2011. Both the machine and the detector are on schedule for a start of commissioning in 2015. The aim of the new facility is to collect a $\Upsilon(4 \mathrm{~S})$ data set equivalent to $50 \mathrm{ab}^{-1}$ by the year 2022. This corresponds to about 55 billion $\Upsilon(4 S) \rightarrow B \bar{B}$ and about 47 billion $e^{+} e^{-} \rightarrow \tau^{+} \tau^{-}$events.

\section{References}

[1] F. Abashian, et al., The Belle Detector, Nucl.Instrum.Meth. A479 (2002) 117-232.

[2] B. Aubert, et al., The BaBar detector, Nucl.Instrum.Meth. A479 (2002) 1-116.

[3] M. Kobayashi, T. Maskawa, CP Violation in the Renormalizable Theory of Weak Interaction, Prog.Theor.Phys. 49 (1973) 652-657.

[4] T. Abe, et al., Belle II Technical Design Report, arXiv:1011.0352 [physics.ins-det].

[5] D. Atwood, M. Gronau and A. Soni, Phys. Rev. Lett. 79 (1997) 185.

[6] B. Grinstein et al., Phys. Rev., D71 (2005) 011504.

[7] W. -S. Hou, Phys. Rev. D 48 (1993) 2342.

[8] T. Aushev, W. Bartel, A. Bondar et al., arXiv:1002.5012 [hep-ex].

[9] W. Altmannshofer, A. J. Buras, D. M. Straub, and M. Wick, JHEP 0904 (2009) 022.

[10] W.J. Marciano and A.I. Sanda, Phys. Lett. B 67 (1977) 303; B.W. Lee and R.E. Shrock, Phys. Rev. D 16 (1977) 1444; T.P. Cheng and L.F. Li, Phys. Rev. D 16 (1977) 1425.

[11] P. Raimondi, Status of the SuperB Effort, URL http://www.lnf.infn.it/conference/superb06/ talks/raimondi1.ppt

[12] J. J. Velthuis et al., Nucl. Instrum. Meth. A 579 (2007) 685. 